RESEARCH ETHICS

\title{
Stopping trials early for commercial reasons: the risk- benefit relationship as a moral compass
}

\section{A S Iltis}

J Med Ethics 2005;31:410-414. doi: 10.1136/jme.2004.008771

\begin{abstract}
Correspondence to:
A S Iltis, Center for Health Care Ethics, Saint Lovis University, 221 N. Grand Blvd, Saint Lovis, $M O$ 63103, USA. iltisas@slu.edu

Received 12 March 2004 In revised form

22 June 2004

Accepted for publication

18 June 2004
\end{abstract}

\begin{abstract}
Decisions by industry sponsors to end clinical trials early for commercial reasons have been the subject of controversy. I argue that the principal consideration in assessing these decisions ought to be the way in which the termination would affect the trial's risk-benefit relationship. If there is not yet sufficient benefit to be gained from the study to offset the risks to which participants were exposed and it is expected that important scientific information would be obtained if the trial were continued, early termination constitutes an unethical alteration of the risk-benefit relationship. This violates the grounds on which permission is given to conduct human research, patients consent to participate, and investigators agree to conduct studies. These knowable and avoidable changes in risk-benefit relationship should generally be seen as impermissible.
\end{abstract}

$\mathrm{T}$ here have been numerous examples in the past decade of sponsors discontinuing trials early for commercial reasons. ${ }^{1-4}$ Sponsors do not usually disclose the precise nature of their decisions; they assert only that trials are stopped for commercial reasons (rather than for safety or efficacy concerns). Because the results of a study terminated early are not normally published (see Black et $a l^{3}$ for an exception), it is difficult to obtain substantial information regarding the nature of the study, the conditions under which it was terminated, the number of participants enrolled, the benefits that were anticipated, and the number of other studies that have been terminated early for commercial reasons. The information available typically comes from investigators or others involved in the study. Table 1 summarises some studies terminated early for commercial reasons. This situation has been the subject of commentary and debate in some of the principal medical journals. ${ }^{124-10}$ Most have rightly criticised such decisions, although not all have taken a critical stance. ${ }^{57}$ Many of the criticisms do not isolate what ought to be the principal consideration in the debate over the permissibility of early discontinuation of trials for commercial reasons: does early termination change the risk-benefit relationship of the study such that the risks to which participants were exposed outweigh the benefits? If so, is it permissible for sponsors knowingly to alter the riskbenefit relationship of a study in an unfavourable way?

If the early discontinuation of a trial results in a study in which the risks to which participants were exposed outweigh the benefits of the study because insufficient data were collected to produce generalisable knowledge, then the decision to discontinue a study early constitutes a retrospective altering of the risk-benefit relationship that violates the conditions under which the investigators were permitted to conduct the research. Contributing generalisable knowledge is the ultimate aim of research and often the primary benefit anticipated. Especially in cases in which participants are not expected to benefit directly from taking part, it serves as the basis for justifying a study. Thus, when a study is cut short for commercial reasons before there are sufficient data to produce generalisable knowledge, the risk-benefit relationship becomes unfavourable. This change is knowable and avoidable. One exception to this may be studies in which participants benefited sufficiently to compensate for the risks to which they were exposed. Such studies, even if they did not contribute generalisable knowledge, cannot be accused of exposing persons to potential harms for insufficient benefit.

Decisions to end trials early for commercial reasons should be distinguished from cases in which it is morally obligatory that a sponsor terminate a trial early. When interim data raise significant safety concerns or suggest that one treatment arm clearly has superior results such that the efficacy of a treatment is established, trials must be ended. ${ }^{11}$ Although one may debate what the stopping rules ought to be for safety and efficacy discontinuations, there is agreement that in theory such terminations are morally required. If a sponsor experiences significant financial problems and cannot continue to fund a trial, it may have to discontinue it early. In some cases, it would be dangerous for a sponsor to attempt to continue a trial if it lacked the financial resources to ensure proper monitoring. Failure to do so would place participants at increased risk. In addition, if participants cannot be recruited at a reasonable rate, it may not be feasible to continue a study and thus a sponsor may be obligated terminate it. ${ }^{1}$ ' Failure to do so would expose those enrolled to the risks of research participation without the prospect of the study resulting in sufficient benefit. New information or circumstances may arise either identifying or creating additional risks, making the known risk-benefit relationship unfavourable. This change is unintentional and unavoidable.

I argue here that generally it is unacceptable to terminate a trial early for commercial reasons if there is not yet sufficient benefit to be gained from the study to offset the risks to which participants have been exposed and it is expected that important scientific information would be obtained if the trial were continued. Clinical trials can yield information that is important even if the results do not support the original hypothesis.

Throughout this article, the term "risk-benefit relationship" is used rather than the more common "risk-benefit

Abbreviations: $D S M B$, data safety monitoring board; IRB, institutional review board 
Table 1 Examples of trials terminated early for commercial reasons

\begin{tabular}{ll}
\hline Study & Reason(s) given for early termination \\
\hline $\begin{array}{l}\text { Randomised controlled trial to assess effects of } \\
\begin{array}{l}\text { Pimagedine (aminoguanidine) on early renal disease } \\
\text { in insulin-dependent diabetes in Europe }\end{array}\end{array}$ & $\begin{array}{l}\text { Incapacity to "adequately fund and } \\
\text { monitor" the study }^{\text {'0 }}\end{array}$ \\
& \\
$\begin{array}{l}\text { Randomised controlled trial comparing liposomal } \\
\text { doxorubicin and cyclophosphamide versus epirubicin } \\
\text { and cyclophosphamide in metastatic breast cancer }\end{array}$ & $\begin{array}{l}\text { The "protocol did not meet the strategic } \\
\text { direction in which [the sponsor] wished to } \\
\text { develop liposomal doxorubicin." (quoting } \\
\text { letter to investigators announcing } \\
\text { the termination) }\end{array}$
\end{tabular}

Controlled onset verapamil investigation of cardiovascular end points (CONVINCE): "a large, simple trial to assess the equivalence of verapamil to standard therapy in preventing cardiovascular disease related events. ${ }^{\prime \prime 3}$

Placebo controlled study of a fluvostatin, Lescol, "for primary prevention of hypercholesterolaemia in men and women aged 70-85 in low cardiovascular risk countries."

Prospective reinfarction outcomes in the thrombolytic era cardizem CD trial (PROTECT) ${ }^{5}$
Commercial reasons; decision "did not derive from a recommendation of the data and safety monitoring board, nor from the review of external data from other clinical trials. ${ }^{\prime \prime 3}$

"... it was feared that a similar trial of pravastatin in primary and secondary prevention in the elderly would reach its conclusion before the fluvostatin trial ... and 'the internal and external environment had changed around Lescol."'1 (partially quoting the communication to the steering committee from the sponsor)

Commercial reasons $s^{5}$
Concerns raised in the literature

"... patients had been included in a demanding study, exposing some of them to measurement of glomerular filtration rates using labelled tracers and to the uncertain safety profile of a new drug, for no personal or scientific benefit whatsoever" ${ }^{\prime \prime 10}$

"... the results of the study may never reach enough statistical power to provide a verified scientific answer ... patients ... may have suffered inconveniences or even contributed their lives to a study now stopped for strategic reasons. ${ }^{\prime 4}$

"When stopped, the results were still inconclusive with respect to the prespecified equivalence bounds ${ }^{\prime \prime 3}$

This was the first study evaluating the costs and benefits of the use of a fluvostatin in this patient population; early termination placed persons at risk and inconvenience of study participation for no benefit as there were insufficient data to assess the therapy.

None raised by reporting author ${ }^{5}$ ratio". The terminology employed here highlights the fact that risks and benefits considered as part of evaluating research proposals cannot be adequately quantified. ${ }^{12}{ }^{13}$ Determinations of the relationship between risks and benefits and the extent to which benefits "outweigh" risks are judgements, not calculations. These judgements are value laden-that is, one has to hold that $\mathrm{X}$ is harmful to consider the possibility of $\mathrm{X}$ occurring to be a risk. The risk-benefit relationship must be understood not as a strictly mathematical one but as a value laden and, to some extent, nebulous one. The standard claim that the risks associated with a study must be "outweighed" by the potential benefits must be understood as a framework for judgement and not taken literally. The goal is to ensure that the possibility for harm is not as great as the possibility that individual persons or society will benefit and that the value of the potential benefits is greater than the disvalue of the potential harms. However, there is no standard calculator or scale that can be used to make this assessment objectively. Despite the ambiguity, the concept of a risk-benefit ratio features prominently in the literature and the regulations governing research. Moreover, as discussed below, there is widespread agreement on the general categories of potential harms (risks) that should be considered in evaluating research proposals.

It is important to notice that risk is used in two different ways. One refers to numerical (for example, 1 in 1000) and quasi-numerical (for example, highly likely) assessments of the probability that something undesirable or harmful will occur. The second use refers to the character of possible harms, which includes but is not limited to the probability of an untoward event occurring. When institutional review boards (IRBs) or comparable bodies ask about the risks of a study, they do not want to know merely that there is, for example, a 1 in 1000 chance that "some harm" may occur. They want to know the character of the potential harms. What harms are possible? How likely are they to occur? How much damage would be involved if they occurred? Are the harms short term or long term? What benefits could emerge from the study? How likely is it that the study will yield those benefits (for example, is it sufficiently powered to produce generalisable knowledge?)? These questions reflect what is meant in this article by the risk-benefit relationship, a relationship that is not reducible to numbers but necessarily involves value judgements and general comparisons of value and disvalue, as well as of possible value and possible disvalue.

Despite the value laden nature of judgements regarding the risk-benefit relationship, there is widespread agreement on the categories of risks (potential harms) and potential benefits that are to be considered in the ethical review of research, even though individual cases may be subject to disagreement. Benefits considered by IRBs or comparable bodies include direct medical benefits to participants and the contribution to generalisable knowledge from which society benefits. The potential harms evaluated include both shortterm and long-term physical harms, including death; psychological harms, such as anxiety that may be experienced after learning that one carries a genetic predisposition to a serious disease; social harms, such as the stigmatisation that could result from loss of confidentiality regarding a medical condition; and economic harms, such as loss of employment or insurance because of research participation.

\section{TERMINATING TRIALS EARLY: CRITICISMS OF THE CRITICISMS}

Sponsors appear to be within their contractual rights when they choose to terminate trials early for commercial reasons; research contracts and consent forms often stipulate that sponsors may withdraw individual participants or end trials at any time for any reason. ${ }^{2}$ It may be unrealistic to think that sponsors would agree to relinquish any control over decisions to terminate trials beyond the authority they already give to data safety monitoring boards (DSMBs). Some have argued 
that it would be wrong for them to do so. ${ }^{5}$ Despite the apparent contractual permissibility of decisions to discontinue trials for commercial reasons, these decisions have met with harsh criticism. Although in most cases the critics are right to object to the early termination of trials for commercial reasons, the justifications offered are not always on target.

Some have criticised the premature discontinuation of trials for commercial reasons on the basis of the impact such decisions will have on the future conduct of research-for example, physicians and patients will lose confidence in sponsors and in the future may refuse to take part in trials as investigators and participants, respectively. ${ }^{10}$ Others are concerned with their impact on the patient-physician relationship (for example, patients enrolled in trials conducted by their own physicians will lose confidence in their doctors $^{1}$ ), and on the psychological wellbeing of patients who may be left with: (1) a lingering fear over the safety of a drug they took as part of a discontinued study; or (2) a feeling of having been deceived by parties that had promised to make their research participation meaningful to the community. ${ }^{1}$ One difficulty with these claims is that their validity depends on whether they are empirically true and on whether we can successfully argue that they matter sufficiently to make trial terminations unethical. If one could ensure that in a particular case these outcomes could be avoided, one would be committed to accepting the permissibility of ending a trial early.

Some have cited a debt owed by sponsors to physicians who may have forgone other research opportunities to conduct the trial in question as grounds for making commercially-driven premature terminations impermissible. ${ }^{1}$ One can imagine that some patients may also have forgone participation in another trial. Even if these opportunity costs are real, one would have to demonstrate that they are sufficiently great to require sponsors to continue trials. Moreover, if a sponsor compensated investigators and participants in a way that overcame or reduced the costs, then the opportunity costs may not be sufficiently large to justify the claim that the trial must go on. Such compensation might involve cash payments, priority in conducting a future trial, or arranging for patients to be accepted into other clinical trials if they so desire. If a sponsor could counteract the opportunity costs, then one would be committed to the view that the premature termination of the trial was permissible.

Others have appealed to the Declaration of Helsinki as prohibiting premature terminations for commercial reasons because it requires that that "considerations related to the well-being of the human subjects should take precedence over the interests of science and society." ${ }^{\prime \prime 14}$ To rely on this clause one must first assess the meaning and context of the term "well-being". A literal interpretation of the clause suggests that, if terminating a trial early does not in itself harm participants, then this clause does not make it impermissible to end a trial early for commercial reasons. One could attempt to interpret "well-being" more broadly to include consideration of the risks to which participants were already exposed and the balance between those risks and the benefits of the study thus far. However, the clause is silent on the risk-benefit relationship, and, in addition, exposure to risk is not necessarily a harm in itself. The clause implies only that certain trials may not be ended early for the benefit of science or society, namely those in which a premature termination would actually harm patients.

There also has been an appeal to the "social responsibilities" of sponsors. ${ }^{6}$ This is ambiguous because no account of the content or source of those responsibilities is offered. If one attempted to give such an account, at best, it would most likely be controversial and thus would not move the discussion forward. Moreover, if a sponsor could show that it was ending a trial early for commercial reasons so that it could fulfil other so-called social responsibilities it has, one would be hard-pressed to say it was impermissible to do so.

Related to the social responsibility claim is an appeal to the claim that contracts between sponsors, investigators, and participants are not like other contracts that operate in the business world. Even though the contracts give sponsors the authority to end trials at any time and for any reason, they should not be taken to mean that. ${ }^{6}$ We should recognise that these are like all other contracts in that there are constraints on the conditions under which they can be developed, signed, and enforced, and on what they can imply. Many contracts to conduct research on human participants are framed by government regulations concerning research on humans. Among the most important of these regulatory requirements and standards in the USA are: (1) the requirement that studies be approved by an IRB or similar body before they commence ${ }^{15-17}$; and (2) that, in order to be approved by an IRB, the expected risks of the study must be outweighed by the potential benefits, either to the participants themselves or to society in terms of knowledge gained (refer to section $111 a 2$ in two US Federal Government publications ${ }^{15}{ }^{17}$ ). A favourable risk-benefit relationship is required not only in the USA but also in most countries that have guidelines governing research. For example, the Australian National Statement on Ethical Conduct in Research Involving Humans prohibits the conduct of research studies whose anticipated risks outweigh the expected benefits. ${ }^{18}$ The European Convention on Biomedicine also stipulates that "the risks which may be incurred by [a research participant] are not disproportionate to the potential benefits of the research." ${ }^{19}$

It is in the importance of the risk-benefit relationship and the role of that relationship in making research morally permissible in the first place that we find the key to understanding why it is generally morally impermissible for sponsors to terminate trials early for commercial reasons. Research that is known to have an unfavourable risk-benefit relationship may not be approved and conducted, and this should be understood as a constraint on contracts between sponsors, institutions, and investigators.

\section{RISK-BENEFIT RELATIONSHIP CONSIDERATIONS}

The role of the risk-benefit relationship in the analysis of the early discontinuation of trials for commercial reasons has been mentioned by others, who have said, for example, that

the only justification for the risks and constraints sustained by [participants] is the potential benefit expected ... [and] ethics committees and health authorities approve studies in view of the balance between the usefulness and the risk of the trial. Once the usefulness is lost, the risk becomes unacceptable.'

This problem of premature trial terminations resulting in altered risk-benefit relationships that make the very conduct of research morally impermissible has not been fully explained or defended in the literature; neither have the full implications of the claim been explored.

IRBs may give permission for research to be conducted only if the expected benefits outweigh the anticipated risks, and changes in protocols may be approved only if, among other things, such changes do not lead to a negative riskbenefit relationship. Investigators must agree to conduct only research that is expected to yield benefits that outweigh the risks to which participants are exposed. Knowingly to alter the risk-benefit relationship violates the conditions under 
which the research was permitted to proceed and under which investigators requested participants' consent.

When trials are terminated early, important scientific questions are often left unanswered and, in many cases, little or no useful information can be gleaned from them. If there are not sufficient data to produce generalisable knowledge at the time, a decision is made to terminate the trial, or if the participants themselves have not benefited sufficiently at the time of the decision, the risk-benefit relationship is shifted so that the risks outweigh the benefits of the study. There is thus a knowable and avoidable violation of the conditions under which an IRB gave permission to conduct a trial and people consented to participate. In one sense, to discontinue a trial early does not violate informed consent because participants are told that sponsors may stop trials, withdraw particular participants, or terminate contracts with specific investigators, at any time. They knowingly consent to these conditions, but they consent with the understanding that there are certain rules and obligations governing research, including the specification that research be conducted only if the expected benefits outweigh the anticipated risks. The conditions under which the investigators were permitted to ask for consent and the very context that framed the consent process are violated. This violation is not accidental, as it is in the case of a trial in which unexpected risks, efficacy concerns, or enrolment deficiencies emerge. It is the result of a conscious, wilful, informed decision made by a sponsor. Moreover, participants did not consent to accept foreseeable negative changes in the risk-benefit relationship of the study. When a sponsor contemplates the decision to stop a trial early for commercial reasons, it should be immediately evident to the sponsor that doing so may result in an inability to obtain generalisable knowledge from the trial, thus changing the risk-benefit relationship. This change can be foreseen and avoided, and sponsors know that a condition for research to be approvable is a favourable riskbenefit relationship. In stopping a trial for commercial reasons prior to having sufficient data to make a contribution to the body of scientific knowledge, the balance is shifted by participants being exposed to risks for little or no compensating benefit to themselves or society. Boyd is right to say that the sponsor nullifies the grounds on which the participants gave consent. ${ }^{8} \mathrm{He}$ is also right to point out that such decisions nullify the grounds on which permission was given to conduct the research in the first place.

The risk-benefit relationship requirement frames the context of research. Decisions regarding changes in a protocol must be made within the setting of this requirement. For example, it is not permissible to enrol more participants than approved because an investigator or sponsor thinks that by doing so better data could be obtained. Permission to increase enrolment is contingent, at least in part, on whether the investigator and the sponsor convince the IRB (or other oversight committee) that the benefits to be gained from enrolling additional participants outweigh the risks associated with doing so. If a decision to end a trial early changes the risk-benefit relationship, it would seem that such a decision is the kind of change in protocol that would require IRB review and approval. There may be practical reasons for which the DSMB should review the request to stop a trial early, including the circumstance that DSMB members are more likely to be familiar with the data and could be in a better position to assess whether continuation would be likely to yield useful information. The DSMB could pass its recommendation to IRBs for final approval, or perhaps DSMBs should be given the authority to make these decisions without IRB intervention. In any case, the decision should be contingent upon assessment of the implications the decision would have for the risk-benefit relationship of the study, and this assessment should not be made unilaterally by the sponsor.

As noted earlier, it may be permissible to end a trial early for commercial reasons if sufficient benefit to individual participants has accrued to compensate for the potential harms to which they were exposed. Judgements regarding the benefits participants have experienced should also be subject to IRB or DSMB review, and should not be pronounced unilaterally by a sponsor.

To stop a trial before sufficient data have been collected in order to maintain a positive risk-benefit relationship also violates the conditions under which investigators will have agreed to conduct a trial. Investigators ought not to expose participants unnecessarily to risks or to more risks than necessary to obtain beneficial information, and those who agree to conduct a study generally do so with the understanding and belief that the study is designed in such a way that the anticipated benefits "outweigh" the anticipated risks.

To shift the risk-benefit relationship knowingly violates the conditions under which participants will have given informed consent, IRBs approved a study, and investigators agreed to conduct the study. The contracts that bind investigators and sponsors, in addition to the informed consent documents signed by participants, often stipulate that the sponsor has the right to stop a trial at any time. However, these documents are signed within a regulatory context that does not allow any party knowingly and intentionally to conduct research that has an unfavourable risk-benefit relationship. The contractual authority that sponsors have to stop a trial should be understood as constrained by their obligation not knowingly and intentionally to change the risk-benefit relationship. One way to avoid these violations is to treat decisions as changes in protocol that require IRB or DSMB review and approval. The circumstances in which some trials have been terminated early for commercial reasons, even when a DSMB has argued that they should not be terminated prematurely, highlight the importance of making such decisions non-unilaterally. ${ }^{6}$

\section{CONCLUSION}

To assess the early discontinuation of trials for commercial reasons, we must ask whether it is wrong to change the riskbenefit relationship. It is argued here that IRBs grant permission for research to be conducted, investigators agree to conduct research, and participants consent to take part in research with an understanding that the relationship of known risks to benefits is favourable, and that the protocol will not be changed in ways that knowingly make it unfavourable. As a result, we should understand sponsors' authority to terminate trials early for commercial reasons as constrained by this requirement. If a premature termination will make the risk-benefit relationship of a study unfavourable, it should be treated, prima facie, as impermissible.

Whenever one suggests that perhaps there should be further restrictions on the research process, one has to consider the widespread implications of such actions. A particular concern is that, to withdraw from sponsors' unilateral authority over decisions to continue trials could hurt the research enterprise and result in less biomedical research. This would translate into fewer new drugs and treatments as well as less knowledge of how best to treat various diseases and conditions. Whether removing the authority from sponsors to terminate trials for commercial reasons, or limiting their authority to do so-for example, by treating such decisions as changes in protocol that require IRB or DSMB review and approval-would in fact damage the research enterprise is an empirical question I cannot answer here. Moreover, whether any such damage would 
lead to more harm than good overall would require further study. The moral seriousness of the problem of knowingly altering the risk-benefit relationship of a study so that the risks outweigh the harms, either while a study is being conducted or after it has reached its endpoint, requires further attention. To make such a change knowingly and intentionally when it could be avoided is, prima facie, a violation of the conditions under which human participant research is permissible.

It is reasonable to ask whether IRBs or DSMBs should sometimes permit sponsors to terminate trials early for commercial reasons, even when doing so alters the riskbenefit relationship in a way that typically would be impermissible. There could be cases of a sponsor who was so financially unstable that to continue a trial would result in total collapse. There is reason to hold that exceptions should be made in such cases. A sponsor forced to continue a trial despite being on the verge of financial collapse could expose participants to an increased risk of harm because-for example, monitoring might be handled inappropriately to save money. Moreover, at some point it may be impossible for the sponsor to make payments and patients may end up being charged for their participation. A sponsor who can demonstrate that to continue a trial would be financially devastating should be permitted to terminate it early for commercial reasons, even when doing so makes the riskbenefit relationship unfavourable. This is not to say that proper concern for patient safety could be ignored in such cases. If terminating a trial early would pose significant risk of harm to participants by-for example, withdrawing them from medication too quickly-provision should be made to protect their safety.

One implication of this analysis is that, when sufficient information is gained so as to maintain a favourable riskbenefit relationship, or patients have benefited sufficiently from their participation so that they maintain a positive riskbenefit relationship, it may be morally permissible to terminate trials early for commercial reasons. This is the case even if there could have been even greater benefit had the trial been continued. This analysis does not rule out all early discontinuations of trials for commercial reasons, which is likely to bring criticism from those who seek an argument to show that all such decisions are morally impermissible. I argue, however, that we are not justified in attempting to prohibit all such decisions. The regulatory environment of research seems aimed at upholding the moral obligation to protect human participants from research risks, not at maximising the benefits of biomedical research. Thus we must ensure that the expected benefits of studies outweigh the anticipated risks, not that the maximum benefits are obtained.

\section{ACKNOWLEDGEMENTS}

I would like to thank two anonymous reviewers for their insightful comments.

\section{REFERENCES}

1 Lièvre $M$, Menard J, Bruckert $E$, et al. Premature discontinuation of clinical trial for reasons not related to efficacy, safety, or feasibility. BMJ 2001;322:603-5.

2 Viberti GC, Slama G, Pozza G, et al. Early closure of European Pimagedine trial. Lancet 1997:350:214-5.

3 Black HR, Elliot WJ, Grandits G, et al. Principal results of the Controlled Onset Verapamil Investigation of Cardiovascular End Points (CONVINCE) Trial. JAMA 2003;289:2073-82.

4 Hopf G. Early stopping of trials. Lancet 1997;350:891.

5 Langer A. Early stopping of trials [letter]. Lancet 1997;350:890-1.

6 Psaty BM, Rennie D. Stopping medical research to save money. JAMA 2003;289:2128-31

7 Ashcroft R. Responsibilities of sponsors are limited in premature discontinuation of trials. BMJ 2001;323:53.

8 Boyd K. Early discontinuation violates Helsinki principles [commentary]. BMJ 2001;322:603.

9 Evans S, Pocock S. Societal responsibilities of clinical trial sponsors. BMJ $2001 ; 322: 569-70$.

10 Anonymous. A curious stopping rule from Hoechst Marion Roussel [editorial]. Lancet 1997;350:155.

11 Brody B. The ethics of biomedical research. New York: Oxford University Press, 1998:156.

12 Pochin E. Risk and medical ethics. J Med Ethics 1982;8:180-4.

13 Herxheimer A. Benefit, risk and harm. Australian Prescriber 2001;24(1):18.

14 World Medical Association General Assembly. Declaration of Helsinki: Ethical medical principles for medical research involving human subjects. JAMA 2000;284:3043-5.

15 United States Federal Government. 45CFR46: Code of federal regulations. 2001. http://www.hhs.gov/ohrp/humansubjects/guidance/45cfr46.htm (accessed 30 November, 2004).

16 United States Federal Government. 21CFR50: Protection of human subjects. 1998. http://www.fda.gov/oc/ohrt/irbs/appendixb.html (accessed 11 March, 2004).

17 United States Federal Government. 21CFR56: Institutional review boards. 1998. http://www.fda.gov/oc/ohrt/irbs/appendixc.html (accessed 11 March, 2004).

18 Australian National Health and Medical Research Council. National statement on ethical conduct in research involving humans. 1992. http:// www.nhmrc.gov.au/publications/humans/contents. htm (accessed 11 March, 2004).

19 Council of Europe Convention for the Protection of Human Rights and Dignity of the Human Being with Regard to the Application of Biology and Medicine: Convention on Human Rights and Biomedicine; 1997 Apr 4; Oviedo, Spain. Oviedo: Council of Europe, 1997, chapter 5, article 16. http:// conventions.coe.int/treaty/en/treaties/html/164.htm laccessed 30 November, 2004). 